\title{
Bay Timoun Ou Manje ${ }^{1}$
}

Adapted by Glenda L. Warren ${ }^{2}$

Bay Timoun Manje (kat a douz mwa)

Lèt manman se lèt sa-a timoun yo bezwen jiskan yo gen kat mwa a si mwa.

Bay ti moun lèt manman jiskan yo gen yon lane. Pa bay timoun yo lèt nan

magazen avan yo gen douz mwa.

\begin{tabular}{|c|c|c|}
\hline & Laj Kat a si Mwa & Laj Sis a uit Mwa \\
\hline $\begin{array}{l}\text { Manje pou } \\
\text { Kòmanse }\end{array}$ & $\begin{array}{l}\text { Timoun Sereal } \\
\text { Kòmanse bay ti moun manje ki gen fè } \\
\text { ladann, nan laj sa yo,tankou diri, sereal ki } \\
\text { gen anpil vitamin ladan-yo. Itilize yon } \\
\text { kiyè lè w-ap bay timoun yo manje sereal. } \\
\text { Fè diri a lejè avèk lèt manmanm oubwen } \\
\text { lèt nan bwat. Apre sa ou kapab bay } \\
\text { timoun yo manje ble ke yo vann nan } \\
\text { magazen yo. }\end{array}$ & $\begin{array}{l}\text { Legim ak Fwi } \\
\text { Kòmanse ba li legim ak fwi. Bay timoun } \\
\text { nan yon sèl kalite manje. } \\
\text { Ji ki Fèt ak Fwi ki gen vitamin C } \\
\text { Bay ti moun yo ji nan yon ti gode. }\end{array}$ \\
\hline $\begin{array}{l}\text { Sin lè } \\
\text { Timoun } \\
\text { nan Pare }\end{array}$ & $\begin{array}{l}\text { Timoun ka } \\
\text { - Chita san difikilte } \\
\text { - Kenbe tèt li dwat } \\
\text { - } \quad \text { Retire manje nan kiyè-a avèk lang li } \\
\text { - } \quad \text { Kenbe pifò sereal-la nan bouch li }\end{array}$ & $\begin{array}{l}\text { Timoun nan ka } \\
\text { - } \quad \text { Chita pou kon't li } \\
\text { - } \quad \text { Bat bouch-li pou'l manje } \\
\text { - } \quad \text { Mete manje nan bouch li pou kon't li } \\
\text { - } \quad \text { Kòmanse bwè nan yon gode }\end{array}$ \\
\hline
\end{tabular}

1. This document is FCS 1065-Hai, one of a series of the Department of Family, Youth and Community Sciences, Florida Cooperative Extension Service, Institute of Food and Agricultural Sciences, University of Florida. First published: June 1997. Publication date: January 2003. Reviewed by Jennifer Hillan, MSH, RD, LD/N, coordinator, Educational/Training Programs, Department of Family, Youth and Community Sciences, University of Florida and by Isabel Valentin-Oquendo, MS, RD, LD/N, Department of OB/GYN, University of Florida,. Translated by: Ducrepin Fleurinord. Translation reviewed by: Jeanelie Gilles, MA, ABD, Dept. of Romance Languages \& Literatures. Please visit the EDIS Web site at http://edis.ifas.ufl.edu

2. Adapted from the third edition (1995) of "Eating Right is Basic," developed by Michigan State University Extension. English version adapted for use in Florida by Glenda L. Warren, M.S., R.D., CFCS, associate professor, Extension nutritionist, EFNEP, Family, Youth and Community Sciences, Cooperative Extension Service, Institute of Food and Agricultural Sciences, University of Florida, Gainesville FL 32611.

The Institute of Food and Agricultural Sciences is an equal opportunity/affirmative action employer authorized to provide research, educational information and other services only to individuals and institutions that function without regard to race, color, sex, age, handicap, or national origin. For information on obtaining other extension publications, contact your county Cooperative Extension Service office. Florida Cooperative Extension Service / Institute of Food and Agricultural Sciences / University of Florida / Christine Taylor Waddill, Dean 


\begin{tabular}{|c|c|c|}
\hline & Laj set a dizan & Laj dis a douzan \\
\hline $\begin{array}{l}\text { Manje pou } \\
\text { Kòmanse }\end{array}$ & $\begin{array}{l}\text { Vyann, Poul ak Pwason } \\
\text { Kòmanse ba yo vyann ki mou, poul ak } \\
\text { pwason ki san zo. Pa mete sèl, grès, sik } \\
\text { ou piman nan manje timoun yo. }\end{array}$ & $\begin{array}{l}\text { Manje sou Zel (Yon ti Pase nan } \\
\text { Bouch) } \\
\text { - } \quad \text { Pen griye } \\
\text { - Ti moso legim bouyi, kale, fwi ki } \\
\text { mou. } \\
\text { - Ti kal vyann moulen, poul oubyen } \\
\text { pwason san zo ladan-yo. }\end{array}$ \\
\hline $\begin{array}{l}\text { Sin lè Timoun } \\
\text { nan Pare }\end{array}$ & $\begin{array}{l}\text { Timoun nan ka } \\
\text { - Kraze manje } \\
\text { - } \quad \text { Pran manje pou kon't li pou'l manje }\end{array}$ & $\begin{array}{l}\text { Timoun nan ka } \\
\text { Itilize yon kiyè ak yon gode }\end{array}$ \\
\hline
\end{tabular}

Enfòmasyon sa-yo te kapab parèt gras a konkou Expanded Food Nutrition Education Program, USDA's food Stamp Program, and University of Florida Cooperative Extension Service. Program Food Stamp -la ede moun ki pa genyen anpil lajan jwen asistans nan lavi chak jou. Li kapab ede-w achte manje ki gen anpil kalite nitritif pou yon bon dayèt. Si ou vle gen plis enfòmasyon sou sa, ou kapab rele nimewo sa-a! 800-342-9274 ( ou pap peye pou apèl-sa-a). 